\title{
Spatio-temporal simulation of energy consumption in China's provinces based on satellite night-time light data
}

Hongwei Xiao ${ }^{\mathrm{a}, \mathrm{b}}$, Zhongyu Ma ${ }^{\mathrm{c}, \mathrm{d}}$, Zhifu $\mathrm{Mi}^{\mathrm{e}, *}$, John Kelsey ${ }^{\mathrm{e}}$, Jiali Zheng ${ }^{\mathrm{e}, \mathrm{f}, \mathrm{g}}$, Weihua Yin ${ }^{\mathrm{a}}$, Min Yan ${ }^{\mathrm{a}}$

${ }^{a}$ Economic Forecasting Department, State Information Center, Beijing 100045, China

${ }^{b}$ Beijing Energy Development Research Center, Beijing 102206, China

${ }^{c}$ State Information Center, Beijing 100045, China

${ }^{\mathrm{d}}$ School of Environment \& Natural Resources, Renmin University of China, Beijing 100872, China

${ }^{\mathrm{e}}$ The Bartlett School of Construction and Project Management, University College London, London WC1E 7HB, UK

${ }^{f}$ Academy of Mathematics and Systems Science, Chinese Academy of Sciences, Beijing, 100190, China

g School of Economics and Management, University of Chinese Academy of Sciences, Beijing, 100190, China

\begin{abstract}
Delay in publication of energy statistics prevents a timely assessment of progress towards meeting targets for energy saving and emission reduction in China. This makes it difficult to meet the requirements to rapidly monitor and evaluate energy consumption for each province. In this study, an alternative approach is provided to estimate the energy consumption by using satellite remote sensing data. We develop spatio-temporal geographically weighted regression models to simulate energy consumption of provinces in China based on the Defense Meteorological Satellite Program's Operational Linescan System (DMSP/OLS) global stable night-time light data. The models simulate China's energy consumption accurately with the goodness of fit higher than $99 \%$. Generally, the national average annual energy consumption is 2.8 billion tonnes of coal equivalent in China between 2000 and 2013, which is close to the actual value with errors smaller than $0.1 \%$. From both temporal and spatial dimensions, the relative errors are smaller than 5.5\% at the provincial level. Therefore, the use of satellite night-time light data provides a useful reference in monitoring and assessing provincial energy consumption in China.
\end{abstract}

Keywords: night-time light data; energy consumption; spatio-temporal geographically weighted regression; China; simulation 


\section{Introduction}

\subsection{Context}

The ability of China to control its energy consumption and carbon emissions is critical not only for China but indeed for the whole planet. The Chinese government attaches great importance to sustainable development, energy conservation, and emission reduction [1-3]. The "Revolution of Energy Production and Consumption (2016-2030)" issued by the National Development and Reform Commission and the National Energy Administration of the People's Republic of China sets a clear target for energy consumption. By 2020, the total energy consumption in China must be controlled within 5 billion tonnes of coal equivalent; $\mathrm{CO}_{2}$ emissions intensity of GDP (the ratio of total $\mathrm{CO}_{2}$ emissions to GDP) should be reduced by $18 \%$ compared to those in 2015 ; and the energy consumption intensity of GDP (the ratio of total energy consumption to GDP) should be reduced by $15 \%$ compared to that in 2015 . By 2030 , the total energy consumption in China must be controlled; China's total annual energy consumption should not exceed 6 billion tonnes of coal equivalent; $\mathrm{CO}_{2}$ emissions intensity of GDP should fall by $60 \%$ $65 \%$ compared with 2005 ; the peak of $\mathrm{CO}_{2}$ emissions should be around 2030 and the targets should be achieved as soon as possible. Looking ahead to 2050, the Chinese economy should aim to produce less than half its energy requirements from fossil fuels. Simultaneously, all provinces have included the total energy consumption control target in the "13th Five-Year Plan" energy-development planning goals. Among these provinces Beijing, Inner Mongolia, Hunan and others regard total energy consumption limits as a binding target [4]. This shows that the Chinese government is fully committed to achieve a controlled reduction of its emissions through setting and maintaining achievable targets [5-7]. However, current delays in the production of China's energy statistics prevents a timely assessment of progress in meeting the targets for energy-saving and emission reduction for each province. Typically data used to update the China Energy Statistical Yearbook is one year out of date and that for the Local Statistical Yearbook around 9-10 months [8, 9].

With the rapid development of satellite remote sensing technology in China, for example, the successful launch of China's first carbon satellite on December 22, 2016, satellite imagery data is continuing to be enriched. There is now a significant possibility for using satellite remote sensing data for reasonably accurate estimation of energy consumption in China's provinces on a sound 
scientific basis in response to which further targets for energy-saving and emission-reducing policies can be formulated according to the observed spatio-temporal changes in provincial energy consumption. This is now in need of urgent research given the rapid progress in satellite-based remote-sensing big data. Global night-time light data acquired by the OLS sensor on the US military meteorological satellite DMSP is an ideal data source for monitoring the intensity of human activities to which energy consumption is closely related. The Defense Meteorological Satellite Program's Operational Linescan System (DMSP/OLS) global night-time light data can be used to estimate energy consumption effectively. By considering regional spatial heterogeneity and comprehensively using global night-time light data together with energy consumption, population size, land area and other statistical data, this paper establishes a spatio-temporal geographically weighted regression model of energy consumption in China based on DMSP/OLS night-time light data which can be used to estimate provincial energy consumption and provide a supplementary reference for the use of satellite remote sensing image data for monitoring and assessment of provincial energy consumption.

\subsection{Successful applications to date}

Sensors to capture global satellite night-time light data are different from sensors by monitoring features of total solar radiation, scattered radiation, direct radiation and reflected radiation, which uses an optical multiplier tube at night with strong photoelectric amplification to effectively detect the low-intensity night-time lights produced by a city's night-time lights and even small-scale residential areas as well as traffic flows. Therefore, the DMSP/OLS night-time light image can be used as a representation of human energy activities and is an ideal data source for monitoring human production and everyday energy use. Since the 1980s, with the gradual improvement of DMSP/OLS global night-time light image data, an increasing number of scholars have applied night-time light data in urbanization monitoring, economic growth assessment, $\mathrm{CO}_{2}$ emissions spatial distribution analysis and energy/electricity consumption estimation, more and more applied research appears, but there are relatively fewer applied studies on provincial energy consumption estimation in China.

In the field of urbanization monitoring, due to variations in atmospheric conditions and periodic changes in satellite sensors, DMSP/OLS night-time light data obtained in different years cannot be directly compared and this makes it difficult to use the time series DMSP/OLS 
night-time light data for urbanization monitoring. Some techniques such as normalizing time series DMSP/OLS night-time light data and deriving urban detection threshold using Pseudo Invariant Features [10] and systematically correcting multi-year multi-satellite night-time stable lights data [11] etc have been developed. These have been successfully applied to the following: urban growth analysis at Liaoning region in China [10], revealed urban expansion in China [11], estimation of urban indicators for individual Chinese cities [12], urbanization processes and rural transition synthetic analysis in China [13], mapping of urbanization dynamics at regional and global scales[14], monitoring of urbanization in India[15]. In these cases the techniques proved to be accurate and effective.

In the field of economic growth assessment, it is possible to consider night light as an indicator of personal consumption [16], and DMSP/OLS night-time light satellite imagery was applied to estimate the following: provincial economic development level of China [17], GDP estimation at different spatial scales and regional levels [18], and efficiency as an estimator of economic activity [19]. Results show that the DMSP/OLS night-time light data can well reveal economic development in different levels.

In the field of spatial distribution analysis of $\mathrm{CO}_{2}$ emissions, integrating the DMSP/OLS night-time stable light (NSL) data with $\mathrm{CO}_{2}$ emissions data [20], and DMSP/OLS night-time light satellite data was applied to the following: analysis of the spatiotemporal distribution of $\mathrm{CO}_{2}$ emission in China [20], estimation of the carbon dioxide emissions in China [21], assessment of China's city-level $\mathrm{CO}_{2}$ emissions arising from energy consumption [22], estimation of $\mathrm{CO}_{2}$ emissions at urban scales of China [23], estimation of $\mathrm{CO}_{2}$ emissions in East Asian Region [24], development of a global $1 \mathrm{~km} \times 1 \mathrm{~km}$ annual fossil fuel $\mathrm{CO}_{2}$ emission inventory [25], evaluation of the constraints on the spatial structure of $\mathrm{CO}_{2}$ emissions from fossil fuels [26], use of imagery as a tool for global mapping of greenhouse gas emissions [27], creation of a global grid of distributed fossil fuel $\mathrm{CO}_{2}$ emissions [28]. Results show that DMSP/OLS night-time light satellite data with $\mathrm{CO}_{2}$ emissions data are good agreement, and can be extended to the future using updated data.

In the field of energy/electricity consumption estimation, research at the global and national levels first explored the correlation between night-time light intensity and energy consumption in the major global economies. Based on the determination of the correlation between the two above, regression analysis models were established to estimate the energy consumption. Shi et al. [29] 
used the calibrated DMSP/OLS Night-time Stabilized Light (NSL) data to characterize the spatio-temporal dynamics of global power consumption. The results indicated that it was relatively appropriate and accurate to estimate the global power consumption by calibrated NSL data. By using the US night-time light data to study 18 cities in the eastern United States, Welch [30] built a regression model of night-time light data together with population, urban area and power consumption variables, which showed that the estimation of electricity consumption performed well using night-time light data at both national and regional scales. Based on DMSP/OLS global night-time light image data, Elvidge et al. [31] conducted a preliminary regression analysis of the relationship between population, economic activity, power consumption and light intensity in 21 countries around the world and found a strong correlation between light intensity and power consumption, which proved that DMSP/OLS night-time light data could be used to estimate power consumption. Chand et al. [32] used DMSP/OLS night-time light data to study the spatio-temporal representation of India's power consumption patterns from 1993 to 2002 and found that night-time light intensity had a significant correlation with power consumption, in which the model's goodness of fit was 0.56. Amaral et al. [33] applied DMSP night-time satellite data to estimate energy consumption in Brazil's Amazon Basin and provided strong evidence for the use of DMSP/OLS night-time satellite sensor images to assess human activities in the Amazon region of Brazil. Letu et al. [34] studied the correlation between DMSP/OLS night-time light intensity and energy consumption in 12 Asian countries including Japan, China, India, etc. and found that the use of polynomial (cubic) regression provides a better estimator of energy consumption. Townsend et al. [35] proved the strong correlation between night-time light and electricity consumption by measuring the spatial distribution of power in Australia with DMSP/OLS night-time light data. He et al. [36] simulated the spatio-temporal dynamics of electricity consumption in mainland China by saturation-corrected DMSP/OLS night-time light data. The results indicated that the average goodness of fit was 0.93 . And the average relative error of electricity consumption in sub regions of mainland China was $-28.9 \%$ in 2000, which demonstrated that the night-time light data could be used to effectively estimate China's electricity consumption.

Research at the provincial and urban levels in China is similar to that at the global and national levels, which mainly establishes efficient inverse models to estimate energy consumption at the 
provincial and urban levels based on global night-time light imagery and energy consumption data. However, there are few applied studies on the estimation of energy consumption in the provinces of China. At present, the night-time light data are mainly used to estimate carbon emissions from energy consumption. Based on global night-time light imagery and energy statistics, Meng et al. [23] proposed a top-down approach to estimate urban carbon emissions in China, which found that night-time light data were suitable for estimating carbon emissions in Chinese cities. According to DMSP/OLS night-time light image data, Su [22] developed a set of remote-sensing assessment methods for China's urban-grade carbon emissions from energy consumption, which solved the problem of the lack of city-level energy consumption statistics in China and the inconsistency in country-province-city data. Besides, carbon emissions of 30 provinces and 66 prefecture-level cities from 1992 to 2010 were simulated by Su [22], with the maximum difference by 269.982 million tonnes between the simulated value and statistical data, the minimum difference by 60,000 tonnes, the mean square error by 9.438 million tonnes, and the relative error by $7.7 \%$. Shi et al. [18], by integrating global night-time stability lighting data and carbon emission statistics, proposed a spatio-temporal carbon emission dynamic model with higher resolution for China. The evaluated results in model indicated that there was a significant positive correlation between China's stable night-lighting data and carbon emission statistics from 1997 to 2012, which might be suitable for estimating carbon emissions below $1 \mathrm{~km}$ resolution.

The above studies have proved that there is a linear correlation between DMSP/OLS global night-time light data and energy consumption at multi-scales involving global, national, provincial and urban levels, thus demonstrating the feasibility of using night-time light data for energy consumption estimation. However, there are a few studies on the use of global night-time light data to simulate energy consumption in China, most of which are linear regression models between the brightness data of night-time light and energy consumption in the preliminary research stage still. Without considering the spatial and temporal differences between the brightness of nightlight data and model parameters of energy consumption among different regions at different times, the goodness of fit of the model and the effect of the simulation still needs to be improved.

With the changes in the world economic structure, the international energy-consumption pattern also shows significant variability. As a major energy consumer in the world, China is 
undergoing profound changes in both total energy consumption growth and diversity of spatial patterns. Taking the provincial energy consumption in China as the research object, this paper uses the spatio-temporal geographically weighted regression (GTWR) to establish the simulation model of provincial energy consumption based on DMSP/OLS night-time light data. Full consideration of the spatio-temporal differences of satellite night-time light intensity and energy consumption at the provincial level in China, can provide a reference for the use of satellite remote sensing data to establish a global-national-regional energy consumption monitoring system as a new "three-dimensional" complementary metric.

\section{Data Sources and Processing}

\subsection{Data sources}

The DMSP/OLS night-time light data used in the study is sourced from the National Geophysical Data Center (NGDC) website of National Oceanic and Atmospheric Administration (NOAA). The latest data published by this website is 22-year global night-time light image data from 1992 to 2013, detected and acquired by six generations of satellite sensors F10, F12, F14, F15, F16, and F18. Different satellites are responsible for detecting global night-time light image data in different periods, including the No. 10 DMSP satellite F10 for 1992-1994, F12 for 1994-1999, F14 for 1997-2003, F15 for 2000-2007, F16 for 2004-2009, and F18 for 2010-2013. There are four types of images in the products of DMSP/OLS night-time light data which can be downloaded, including cloudless observation frequency, average light, stable light, and average visible light.

Among the four types of images above, there is a close relationship between the quality of the cloudless observation frequency image data and the number of observations. The less the number of observations, the lower the quality of data; conversely, the more, the higher. In terms of the average light image data, it still contains noise such as short-lived light sources without excessive noise reduction processing. While the stable light image data is of higher quality after processing for flares and accidental noises, whose DN values ranges from 0 to 63 . Although the average visible light image data considers the percentage of incidental noise frequencies in the light observation period, which could reduce the influence of flare and accidental noise to a certain extent, it is still unable to remove most of the flare and accidental noise with relatively frequent detections. Comparing the effect on the removal of flare and accidental noise by four types of 
DMSP/OLS night-time light data products, this study selects stable light image data as the night-time light intensity variable for the spatio-temporal simulation of energy consumption by provinces.

The energy consumption data used in this paper is obtained from the "China Energy Statistical Yearbook" and the provincial statistical yearbooks. The data from China Energy Statistics Yearbook 2014 indicates that the National Bureau of Statistics of China has adjusted the energy statistics according to the results of the third economic census. Correspondingly, the energy consumption data of 30 provinces and cities were also adjusted in 2013, while the data of Tibet was not available. However, the provincial energy consumption data before 2013 has not been adjusted yet, and only the total energy consumption since the year 2000 has undergone adjustments. Consequently, the energy consumption data of provinces in 2013 and later is based on the latest "China Energy Statistical Yearbook". And the provincial energy consumption data before 2013 comes from the statistical yearbooks of the provinces, with some adjustments according to the third national economic census data. ${ }^{1}$

\subsection{Data processing}

Since the DMSP/OLS night-time light data was acquired by both new and old generations of satellite sensors simultaneously in some years in the period 1992-2013 (F10 and F12 for 1994, F12 and F14 for 1997-1999, F14 and F15 for 2000-2003, F15 and F16 for 2004-2007), the image data acquired by both generations in 1994 and 1997-2007 is compared, which shows huge differences of data by two sensors in the same year. To improve the depiction of satellite night-time light image data for China's provincial energy consumption, the method of Liu et al. [11] is applied for inter-calibration, intra-annual integration and inter-annual correction of the DMSP/OLS stable night-time light image data between 1992 and 2013. For inter-calibration, the first step is to select the Jixi City in Heilongjiang Province as the reference area for image pixels to be calibrated, for it is relatively stable in economic and social development from 1992 to 2013. Second, the night-time light image detected by the 16th DMSP satellite F16 in 2007 is selected as a reference image for correcting stable light images. Third, a quadratic polynomial regression

\footnotetext{
${ }^{1}$ More particularly, based on the results of the third national economic census, Beijing, Heilongjiang, Jiangsu, Gansu and Qinghai adjusted the energy consumption data for 2005-2012; Hebei, Shanxi, Anhui, Hainan and Shaanxi adjusted data for 2010-2012; Tianjin, Shanghai, Zhejiang, Fujian, Jiangxi, Henan, Guangxi, Ningxia and Xinjiang adjusted data after 2000; while there has been no adjustment by Inner Mongolia, Liaoning, Jilin, Shandong, Hubei, Hunan, Guangdong, Chongqing, Sichuan, Guizhou and Yunnan.
} 
model $D N_{\text {correct }}=\alpha \times D N^{2}+\beta \times D N+\gamma$ is chosen to construct a correction equation. The parameters $\alpha, \beta, \gamma$ of different satellite correction equations in different years are obtained by comparing the DN value of stable night-time light intensity detected by satellites of the current year and the stable night-time light intensity DN value of Jixi City detected by No. 16 DMSP satellite F16. The fourth step is to use the quadratic polynomial regression model and the corresponding correction equation's empirical parameters to correct stable night-time brightness DN values each year. For intra-annual integration, the night-time light image elements acquired by the new and old generations of satellite sensors in 1994 and 1997-2007 are compared. If the image pixel has only brightness in one of the satellite images, the image element is marked as unstable pixel and accordingly merges its $\mathrm{DN}$ value to 0 during the year. If the image pixel has brightness in both old and new generations of satellite images, the image pixel is marked as a stable pixel and the DN value is accordingly fused into the average of the DN values of the old and new generations of satellite image pixels. For inter-annual correction, the same image element is compared during the inter-annual period. If the image pixel has brightness only in the earlier years, the image pixel is marked as an unstable pixel and the DN value is interval corrected as 0 . If the image pixel has brightness during the detection period, the image pixel is marked as a stable pixel and it is necessary to ensure that the DN value of the earlier image pixel does not exceed the DN value of the later image pixel.

From 2000 to 2016, there were statistical changes in the energy consumption data of China's sub-provinces around 2013. According to the results of the third economic census, the National Bureau of Statistics of China adjusted the energy consumption data of the whole country and sub-provinces. Before the spatio-temporal simulation of energy consumption in China based on satellite night-time light data, it is necessary to adjust the provincial energy consumption data from 2000 to 2012. According to the analysis of provincial energy consumption data from 2013 to 2016, the provincial energy consumption accounts for a relatively stable proportion of the national energy consumption, and its proportionatel changes from 2014 to 2016 are relatively stable. Therefore, this article adjusts the energy consumption of unadjusted provinces in unadjusted years through the percentage estimation. By assuming that the change in the proportion of provincial energy consumption accounting for national energy consumption in 2013 is the average of 
2014-2016, the change of proportion in 2001 would be the average of 2002-2004 by analogy. Thus, the proportion of energy consumption in each province to that in the country during 2000-2012 could be estimated by the change of proportion during 2001-2013. To make full use of the adjusted data in the third economic census, the adjustment is divided into three periods involving 2010-2012, 2005-2009 and 2000-2004.

In the period of 2010-2012, 19 provinces adjusted the results according to the third economic census $^{2}$. The adjustment process of energy consumption data for the remaining 11 provinces in 2010-2012 is as follows: first, Hebei, Shanxi and Xinjiang, whose percentages change more than $0.1 \%$, are eliminated to avoid a large impact on aggregate total energy consumption of sub-provinces caused by changes of proportions, and the total energy consumption then could be estimated by the adjusted data and proportions of remaining 16 provinces; second, the estimated average of total energy consumption is selected as the aggregate total energy consumption of sub-provinces by 16 provinces; finally, the energy consumption in 11 provinces is measured by the estimated total energy consumption and the proportion of remaining 11 provinces. The estimation results demonstrate that the total energy consumption of sub-provinces are 36.2, 39.1 and 4.09 billion tonnes of coal equivalent in 2010, 2011 and 2012, respectively.

Similarly in the period of $2005-2009,14$ provinces adjusted data according to the results of the third economic census ${ }^{3}$. The adjustment process of energy consumption data for the remaining 16 provinces in 2005-2009 repeats three steps above: first, Xinjiang and Tianjin with severely low energy consumption are eliminated, leaving the remaining 12 provinces for estimation; second, the average is selected as the total for 12 provinces; finally, both estimated total and proportion are used for the remaining 16 provinces. The estimation results indicate that the total energy consumption of sub-provinces are 26.7, 29.0, 31.5, 33.2 and 3.47 billion tonnes of coal equivalent in 2005, 2006, 2007, 2008 and 2009, respectively.

However in the period of 2000-2004, only nine provinces adjusted data according to the results of the third economic census ${ }^{4}$. By eliminating Tianjin with estimation results significantly lower than the national level, Jiangxi and Ningxia with higher estimation results, and Xinjiang

219 provinces including Beijing, Tianjin, Hebei, Shanxi, Heilongjiang, Shanghai, Jiangsu, Zhejiang, Anhui, Fujian, Jiangxi, Henan, Guangxi, Hainan, Shaanxi, Gansu, Qinghai, Ningxia and Xinjiang.

314 provinces including Beijing, Tianjin, Heilongjiang, Shanghai, Jiangsu, Zhejiang, Fujian, Jiangxi, Henan, Guangxi, Gansu, Qinghai, Ningxia, and Xinjiang.

${ }^{4}$ Nine provinces including Tianjin, Shanghai, Zhejiang, Fujian, Jiangxi, Henan, Guangxi, Ningxia, and Xinjiang. 
changing more than $0.1 \%$, samples of the remaining five provinces are too small to be used for estimation. By comparing the total energy consumption of sub-provinces and the national total energy consumption in 2005-2016, the former exceeds the later by less than 5\%. Thus, the provincial energy consumption could be calculated based on a reasonable estimation of aggregate total energy consumption of sub-provinces in 2000-2004. The adjustment process of energy consumption data for the remaining 21 provinces in 2000-2004 is as follows: first, by assuming that the ratio of provincial total energy consumption to national total energy consumption in 2014 is the average of 2005-2007, the ratio in 2000 would be the average of 2001-2003 by analogy; second, by the ratio of provincial total energy consumption to national total energy in 2000-2004 and the national total energy consumption data, the total energy consumption of sub-provinces in 2000-2004 is estimated; finally, both estimated total and proportion are used for the remaining 21 provinces. The estimation results show that the total energy consumption of sub-provinces are 15.3, 16.2, 17.6, 20.3 and 2.36 billion tonnes of coal equivalent in 2000, 2001, 2002, 2003 and 2004, respectively.

As the conversion factors are various, the sum of provincial energy consumption data is not equal to the national total. To compare the provincial spatio-temporal simulation results with the national total, the annual energy consumption forecast value is converted by the ratio of provincial total energy consumption to national total energy consumption based on the simulation of provincial energy consumption by a spatio-temporal geographically weighted regression model.

\section{Simulation Model}

In China, types and structures of provincial energy consumption vary caused by different factors involving resource endowment, population size, economic development, industrial structure, and technological level with a vast territory. Hence, there are also great spatial and temporal differences in the energy consumption of provinces. To simulate the energy consumption of China's sub-provinces more scientifically and accurately, this study chose per capita energy consumption and energy consumption per unit area as the research variables to eliminate the impact of the scale factor. First, two spatio-temporal geographically weighted regression models are constructed, by per capita energy consumption with stable night-time light intensity DN (DN/grid) as Model 1, and energy consumption per unit area with stable night-time light intensity DN (DN/grid) as Model 2, respectively. Second, the DN value of the stability night-time light 
brightness is used to space-simulate and time-simulate the per capita energy consumption and energy consumption per unit area in provinces. Finally, the provincial energy consumption is estimated by the population size and land area.

To fully investigate the heterogeneity of stable night-time light DN and spatial spillover effects in different provinces, a spatio-temporal geographically weighted regression model is constructed including spatial lags for stable night-time brightness DN values as follows:

$$
\begin{aligned}
& \ln P E C_{i t}=\alpha_{0}\left(u_{i}, v_{i}, t_{i}\right)+\alpha_{1}\left(u_{i}, v_{i}, t_{i}\right) \ln D N_{i t}+\alpha_{2}\left(u_{i}, v_{i}, t_{i}\right) W^{*} \times \ln D N_{i t}+\varepsilon_{i t} \\
& \ln S E C_{i t}=\beta_{0}\left(u_{i}, v_{i}, t_{i}\right)+\beta_{1}\left(u_{i}, v_{i}, t_{i}\right) \ln D N_{i t}+\beta_{2}\left(u_{i}, v_{i}, t_{i}\right) W^{*} \times \ln D N_{i t}+\varphi_{i t}
\end{aligned}
$$

In Eq. (1), $P E C_{i t}$ represents the per capita energy consumption of the provincial region $i$ in the year $t ; S E C_{i t}$ refers to the energy consumption per unit area of the provincial region $i$ in the year $t ; D N_{i t}$ refers to the stable night-time lightness $D N$ of the provincial region $i$ in the year $t$; and $W^{*}$ is a spatial weight matrix that combines geographical and economic information; $\left(u_{i}, v_{i}\right)$ is the longitude and latitude of the provincial region; and $\alpha_{0}\left(u_{i}, v_{i}, t_{i}\right)$ and $\beta_{0}\left(u_{i}, v_{i}, t_{i}\right)$ are the interceptions of the spatio-temporal geographical weighted regression model among the per capita energy consumption and energy consumption per unit area to the stable night-time brightness $D N$ of the provincial region $i$ in the year $t ; \alpha_{1}\left(u_{i}, v_{i}, t_{i}\right)$ and $\beta_{1}\left(u_{i}, v_{i}, t_{i}\right)$ are the regression coefficients of the light intensity $D N$ value for the per capita energy consumption and energy consumption per unit area for the provincial level $i$ in the year $t ; \alpha_{2}\left(u_{i}, v_{i}, t_{i}\right)$ and $\beta_{2}\left(u_{i}, v_{i}, t_{i}\right)$ are the regression coefficients of the spatial spillover effects of light intensity $D N$ value for the per capita energy consumption and energy consumption per unit area for the provincial level $i$ in the year $t$; and $\varepsilon_{i t}, \varphi_{i t}$ are the residual items of the provincial region $i$ in year $t$ for Model 1 and Model 2.

Spatial weight matrix $W^{*}=W^{G S} \times E$, which contains both geographic and economic information, is the point multiplication of geographical and economic spatial weight matrix. The geographical spatial weight matrix is inversely proportional to the large circle distance; the formula is as follows: 


$$
W_{i j}^{G S}=\left\{\begin{array}{cc}
\frac{1}{\left\{R \times \arccos \left[\sin x_{i} \sin x_{j}+\cos x_{i} \cos x_{j} \cos \left(y_{i}-y_{j}\right)\right]\right\}^{\alpha}} & i \neq j \\
0 & i=j .
\end{array}\right.
$$

In Eq. (2), $R \times \arccos \left[\sin x_{i} \sin x_{j}+\cos x_{i} \cos x_{j} \cos \left(y_{i}-y_{j}\right)\right]$ is the great-circle distance of the two spatial positions $A_{i}\left(x_{i}, y_{i}\right)$ and $A_{j}\left(x_{j}, y_{j}\right), R$ is the radius of the earth; $x, y$ represents latitude and longitude of geospatial location; $\alpha$ is an appropriate parameter, typically 1 or 2 , and has a value of 2 in this paper; the corresponding spatial weight is the square of the distance reciprocal.

The economic spatial weight matrix uses the similarity of the economic development level between spatial location units to reflect the adjacent spatial non-geographical elements.

$$
E_{i j}=\left\{\begin{array}{cc}
\frac{1}{\left|\bar{G}_{i}-\bar{G}_{j}\right|^{\alpha}+m} & i \neq j \\
0 & i=j
\end{array}, \quad \bar{G}_{i}=\frac{1}{14} \sum_{t=2000}^{2013} G_{i t} .\right.
$$

In Eq. (3), $G_{i t}$ is the per capita GDP, which represents the economic development level of the year $t$ in the region $i$ (based on the constant price in 2000); $\alpha$ is the adjustment parameter of the economic weight. Normally, it takes a value of 1 or 2 , and the value is 2 in this paper. When the per capita GDPs of two different regions in the same time period are equal, the denominator is 0 . So, if the per capita GDPs of any two different regions in the same time period are equal, $\mathrm{m}=1$. If any two different regions have different per capita GDPs in the same time period, $\mathrm{m}=0$.

The core of the spatio-temporal geographically weighted regression model is the space-time weight matrix, which typically chooses different space-time weight functions to describe the space-time relationship. The space-time weight function in this paper combines the Gaussian function method and space-time distance. The form of space-time weight function is as follows:

$$
w_{i j}^{S T}=\exp \left\{-\left(\frac{d_{i j}^{S T}}{b_{S T}}\right)^{2}\right\}=\exp \left\{-\left(\frac{\alpha\left[\left(u_{i}-u_{j}\right)^{2}+\left(v_{i}-v_{j}\right)^{2}\right]+\beta\left(t_{i}-t_{j}\right)^{2}}{b_{S T}{ }^{2}}\right)\right\} .
$$

In Eq. (4), $d_{i j}^{S T}=\sqrt{\alpha\left[\left(u_{i}-u_{j}\right)^{2}+\left(v_{i}-v_{j}\right)^{2}\right]+\beta\left(t_{i}-t_{j}\right)^{2}}$ is the space-time distance, $b_{S T}$ is the bandwidth of the space-time weight function; cross-validation is typically used to select the 
optimal bandwidth.

Based on stable night-time light data, China's energy consumption measurement model for sub-provinces is as follows:

$$
\begin{aligned}
& E C 1_{i t}=P_{i t} \times P E C_{i t}=P_{i t} \times \exp \left\{\alpha_{0}\left(u_{i}, v_{i}, t_{i}\right)+\alpha_{1}\left(u_{i}, v_{i}, t_{i}\right) \ln D N_{i t}+\alpha_{2}\left(u_{i}, v_{i}, t_{i}\right) W^{*} \times \ln D N_{i t}\right\} \\
& E C 2_{i t}=S_{i t} \times S E C_{i t}=S_{i t} \times \exp \left\{\beta_{0}\left(u_{i}, v_{i}, t_{i}\right)+\beta_{1}\left(u_{i}, v_{i}, t_{i}\right) \ln D N_{i t}+\beta_{2}\left(u_{i}, v_{i}, t_{i}\right) W^{*} \times \ln D N_{i t}\right\}
\end{aligned}
$$

In Eq. (5), $E C 1_{i t}$ and $E C 2_{i t}$ are the provincial energy consumption of the region $i$ measured in year $t$ through per capita energy consumption and energy consumption per unit area, respectively. $P_{i t}$ and $S_{i t}$ are the population size and land area of provincial level $i$ in year $t$, respectively.

\section{Simulation Results}

\subsection{Variable selection}

The National Bureau of Statistics has revised the energy consumption data since 2000 based on the results of the third national economic census. Taking 2013 as an example, the total energy consumption after revision was increased by 420 million tonnes of coal equivalent. More specifically, the proportion of coal consumption in total energy consumption was increased by $1.4 \%$; oil and gas consumption as a share of total energy consumption decreased by $1.3 \%$ and $0.5 \%$, respectively; and the proportion of primary electricity and other energy to total energy consumption increased by $0.4 \%$. The increase in the total energy consumption and the share of coal consumption has made a greater impact on the basic data of energy consumption. To be consistent with the revised energy statistics data, this study selects the provincial energy consumption data since 2000 as the research object. The latest DMSP/OLS data released global night-time light image data for 22 years from 1992 to 2013. Consequently, China's provincial energy consumption spatio-temporal simulation is studied by the satellite night-time light data from 2000 to 2013. Night-time stable light data comes from the National Geophysical Data Center website of the NOAA of the United States. The energy consumption data is obtained from the "China Energy Statistical Yearbook" and the statistical yearbooks of the provinces. The data on the population size and land area of provinces is derived from "China Statistical Yearbook".

\subsection{General simulation results}

On the basis of obtaining the DN values of DMSP/OLS stability at night in 30 provinces by 
inter-calibration, intra-annual integration and inter-annual correction, the influential coefficients of DN at steady night-time light intensity and its spatially lagging term on the per capita energy consumption and energy consumption per unit area in different provinces are estimated by a spatio-temporal geographically weighted regression model. Through cross-validation, the optimal bandwidths of Model 1 and Model 2 are determined to be 0.2518 and 0.2415 , respectively. The overall results of two models perform well, in which the goodness of fit are as high as $99.74 \%$ and 99.95\%, respectively. According to the description statistics of parameters estimation by two models in Tables 1 and Table 2, the DN value of the stable night-time light intensity and its spatially lagging term have a greater variation in the influential coefficient both by the per capita energy consumption and the energy consumption per unit area, which further indicates that the spatial and temporal heterogeneity of the satellite night-time light data need to be considered when simulating the energy consumption of China's provinces. This further proves that it is more practical to choose a spatio-temporal geographically weighted regression model to capture the temporal and spatial dynamic characteristics of provincial energy consumption.

Based on using satellite stabilized night-time light data to stimulate provincial energy consumption in China per capita energy consumption and energy consumption per unit area, indirect energy consumption is calculated according to population size with per capita energy consumption, and land area with energy consumption per unit area in each region. The simulation results show that the simulated value and actual value of average annual energy consumption are similar between 2000 and 2013, and the relative errors of the two models are both within $0.1 \%$. Among them, the annual average energy consumption simulated by Model 1 in 2000-2013 is $2.8433 \times 10^{9}$ tce, which is close to the actual annual average energy consumption of $2.8444 \times 10^{9}$ tce. The absolute error is $-1.1165 \times 10^{6}$ tce, and the relative error is $0.04 \%$. The average annual energy consumption simulated by Model 2 in 2000-2013 is $2.8457 \times 10^{9}$ tce, which is also similar to the actual annual energy consumption of $2.8444 \times 10^{9}$ tce. The absolute error is $1.2824 \times 10^{6}$ tce, and the relative error is $0.05 \%$.

Table 1 Description statistics of parameter estimation in Model 1 (optimal bandwidth $=0.2518)$

\begin{tabular}{ccccccc}
\hline Variable & Minimum & First Quartile & Median & Third Quartile & Maximum & Interquartile range \\
\hline constant & -6.7660 & 1.8489 & 3.6450 & 4.7317 & 9.0110 & 2.8828
\end{tabular}




\begin{tabular}{ccccccc}
$\ln (D N)$ & -5.2288 & -0.1400 & 0.5304 & 1.8060 & 7.3183 & 1.9460 \\
$W^{*} \times \ln (D N)$ & -2.6984 & -0.08276 & 0.4307 & 1.6291 & 6.0901 & 1.7119 \\
\hline Table 2 Description statistics of parameter estimation in Model 2 (optimal bandwidth = 0.2415) \\
\hline Variable & Minimum & First Quartile & Median & Third Quartile & Maximum & Interquartile range \\
\hline constant & -10.3374 & 2.5664 & 3.9663 & 4.7301 & 8.0755 & 2.1637 \\
$\ln (D N)$ & -4.7315 & 0.3688 & 1.4038 & 2.1683 & 8.1519 & 1.7995 \\
$W^{*} \times \ln (D N)$ & -2.7616 & -0.3140 & 0.2588 & 1.3054 & 6.6377 & 1.6194 \\
\hline
\end{tabular}

\subsection{Temporal and spatial simulation results}

From both time and space perspectives, the spatio-temporal simulation of energy consumption in China based on satellite night-time light data perform well.

Table 3 shows the comparison on the simulation effect of total energy consumption from 2000 to 2013. From a time perspective, the relative errors in all years are within 5.5\%. In 2006, the simulated provincial energy consumption is the closest to the actual value, and the absolute error and relative error are both small. The simulated values of energy consumption for Model 1 and Model 2 are $2.8665 \times 10^{9}$ tce and $2.8739 \times 10^{9}$ tce, respectively, which are very close to the actual value of 2.8647109 tce in 2006, and the relative errors are only $0.06 \%$ and $0.32 \%$, respectively.

Fig. 2A indicates the comparison on the simulation effect of annual average energy consumption in provinces from 2000 to 2013. From the space perspective, simulated values of average annual energy consumption are very close to actual values in 2000-2013, where relative errors of all provinces are within $1 \%$. A large number of provinces and cities performed well with relative errors within $0.2 \%$ in Model $1^{5}$, similarly, well-performed provinces and cities with relative errors within $0.2 \%$ in Model $2 .{ }^{6}$

Fig. 2B illustrates the comparison on the simulation effect of energy consumption in 2013. From both time and space perspectives, relative errors of provinces in 2013, as an example, are all within 5\% apart from Qinghai and Xinjiang, where relative errors are slightly higher than 5\%. A large number of provinces and cities performed well with relative errors within $0.2 \%$ in both

\footnotetext{
${ }^{5}$ Involving Shanxi, Inner Mongolia, Shanghai, Zhejiang, Anhui, Jiangxi, Shandong, Henan, Hubei, Hunan, Guangdong, Yunnan, Qinghai, Ningxia, Xinjiang, etc.

${ }^{6}$ Include Tianjin, Hebei, Shanxi, Inner Mongolia, Jilin, Heilongjiang, Jiangxi, Shandong, Henan, Hubei, Hunan, Yunnan, Shaanxi, Qinghai, Ningxia, Xinjiang, etc.
} 
Model 1 and Model $2^{7}$, besides, well-performed provinces and cities with relative errors within $1 \%$ in Model 1 include Hebei, Jilin, Chongqing, etc. The provinces who get the smallest absolute error and relative error in Model 1 and Model 2 are Guangxi and Beijing, with relative errors showing $0.06 \%$ and $0.07 \%$, respectively. Their simulated values of the model and the actual energy consumption are also relatively close.

Table 3 Comparison on the simulation effect of total energy consumption from 2000 to 2013

\begin{tabular}{|c|c|c|c|c|c|c|c|}
\hline \multirow[b]{2}{*}{ Year } & \multirow[b]{2}{*}{$\begin{array}{l}\text { Actual Energy } \\
\text { Consumption } \\
\left(10^{4} \text { tonnes of }\right. \\
\text { coal } \\
\text { equivalent })\end{array}$} & \multicolumn{3}{|c|}{ Simulation Results in Model 1} & \multicolumn{3}{|c|}{ Simulation Results in Model 2} \\
\hline & & $\begin{array}{c}\text { Predicted } \\
\text { Value }\left(10^{4}\right. \\
\text { tonnes of } \\
\text { coal } \\
\text { equivalent } \\
\quad)\end{array}$ & $\begin{array}{c}\text { Absolute } \\
\text { Error }\left(10^{4}\right. \\
\text { tonnes of } \\
\text { coal } \\
\text { equivalent })\end{array}$ & $\begin{array}{l}\text { Relative } \\
\text { Error }(\%)\end{array}$ & $\begin{array}{c}\text { Predicted } \\
\text { Value }\left(10^{4}\right. \\
\text { tonnes of } \\
\text { coal } \\
\text { equivalent } \\
\quad)\end{array}$ & $\begin{array}{c}\text { Absolute } \\
\text { Error }\left(10^{4}\right. \\
\text { tonnes of } \\
\quad \text { coal } \\
\text { equivalent })\end{array}$ & $\begin{array}{l}\text { Relative } \\
\text { Error (\%) }\end{array}$ \\
\hline 2000 & 146964.00 & 146288.78 & -675.22 & -0.46 & 146266.04 & -697.96 & -0.47 \\
\hline 2001 & 155547.00 & 154963.76 & -583.24 & -0.37 & 154691.71 & -855.29 & -0.55 \\
\hline 2002 & 169577.00 & 173591.71 & 4014.71 & 2.37 & 172629.48 & 3052.48 & 1.80 \\
\hline 2003 & 197083.00 & 207844.54 & 10761.54 & 5.46 & 207620.82 & 10537.82 & 5.35 \\
\hline 2004 & 230281.00 & 229561.17 & -719.83 & -0.31 & 229523.88 & -757.12 & -0.33 \\
\hline 2005 & 261369.00 & 251912.27 & -9456.73 & -3.62 & 253283.77 & -8085.23 & -3.09 \\
\hline 2006 & 286467.00 & 286648.09 & 181.09 & 0.06 & 287385.65 & 918.65 & 0.32 \\
\hline 2007 & 311442.00 & 305457.88 & -5984.12 & -1.92 & 306272.77 & -5169.23 & -1.66 \\
\hline 2008 & 320611.00 & 324031.75 & 3420.75 & 1.07 & 325020.80 & 4409.80 & 1.38 \\
\hline 2009 & 336126.00 & 334879.39 & -1246.61 & -0.37 & 334025.22 & -2100.78 & -0.62 \\
\hline 2010 & 360648.00 & 363901.73 & 3253.73 & 0.90 & 362756.35 & 2108.35 & 0.58 \\
\hline 2011 & 387043.00 & 387583.58 & 540.58 & 0.14 & 388658.26 & 1615.26 & 0.42 \\
\hline 2012 & 402138.00 & 403116.82 & 978.82 & 0.24 & 404588.75 & 2450.75 & 0.61 \\
\hline 2013 & 416913.00 & 410796.50 & -6116.50 & -1.47 & 411280.89 & -5632.11 & -1.35 \\
\hline Average & 284443.50 & 284327.00 & -116.50 & -0.04 & 284571.74 & 128.24 & 0.05 \\
\hline
\end{tabular}

\footnotetext{
${ }^{7}$ Involving Beijing, Liaoning, Shanghai, Zhejiang, Shandong, Hubei, Hunan, Guangxi, Hainan, Sichuan and
} Guizhou. 

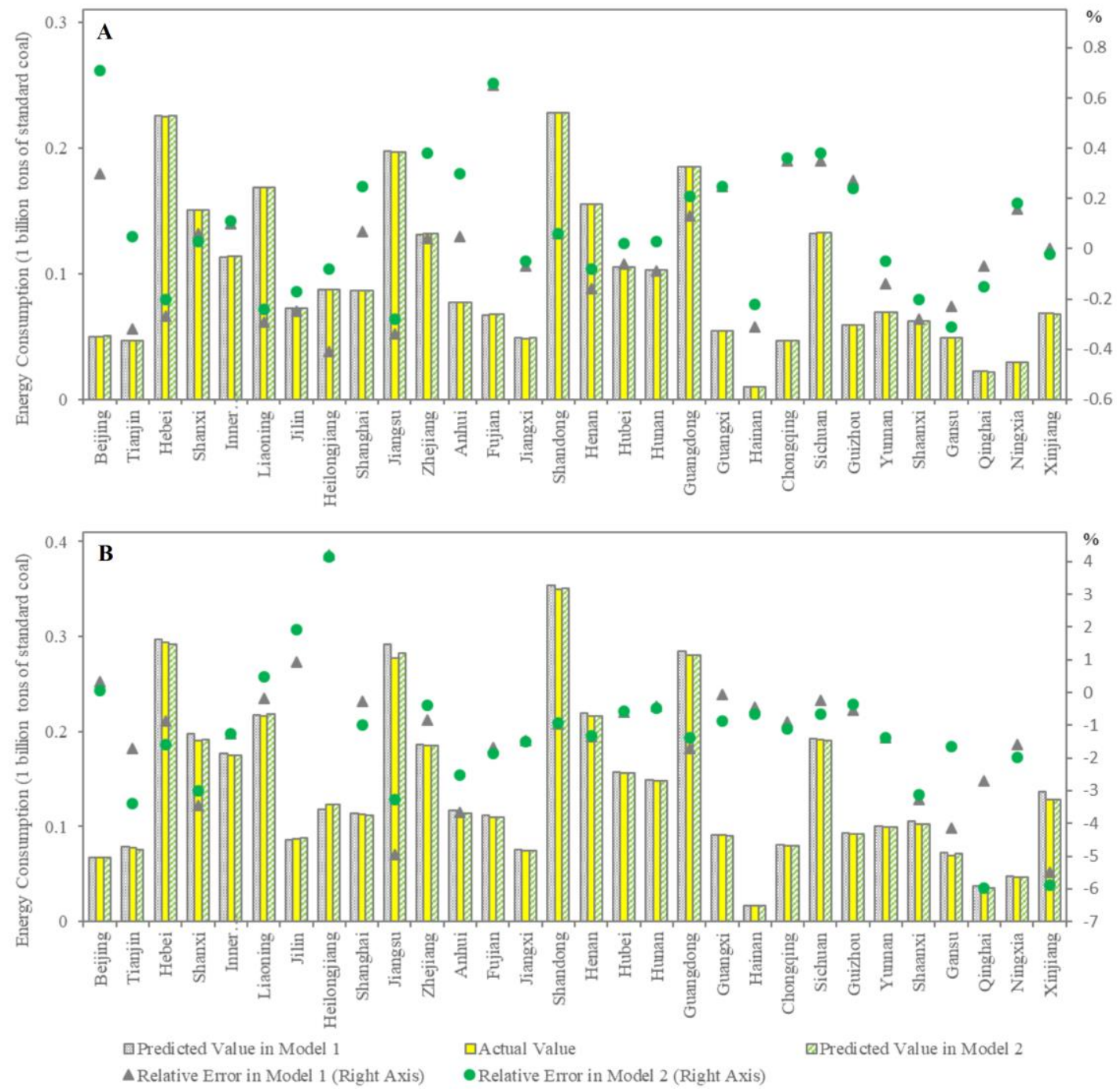

Fig. 2. (A) Comparison on the simulation effect of annual average energy consumption in provinces from 2000 to 2013; (B) comparison on the simulation effect of energy consumption in 2013.

\subsection{Model application}

China's provincial energy consumption spatio-temporal simulation results perform well based on stable night-time light data, indicating that it is feasible to use satellite night-time lighting data to rapidly estimate energy consumption in China's provinces. "China Energy Statistical Yearbook" and "Local Statistical Yearbook" are the important basis of assessments on energy-saving and emission-reducing targets in each province. At present, the data update of the "China Energy Statistical Yearbook" typically lags behind by one year and the data update of the "Local Statistical Yearbook" typically lags behind by 9-10 months, which brings a certain lag to the provincial energy-saving and emission-reducing target assessment and makes it difficult to meet 
the energy saving requirements for rapidly monitoring and evaluating consumption. With the continuous expansion of China's application of high-resolution satellite data, satellite remote sensing has also played unique advantages of all days, three-dimensional, and continuous observation in the fields of energy conservation, emission reduction, and climate change response. One more obvious use is to observe energy consumption changes as a result of extreme weather events which we are told will become more frequent.

We have entered a critical period during which critical changes in global temperatures may be reached causing irreversible damage to the ecosystem. Any means that can be devised to shorten to the time loop from observation to actual observed change through policy is therefore of potentially critical importance. Through constructing a spatio-temporal geographically weighted regression model between satellite remote sensing data and energy consumption, the regression model provides relatively accurate data for provincial energy-consumption monitoring and evaluation, which is an effective complement to the rapid implementation of provincial energy consumption monitoring and assessment in the era of satellite remote sensing. The spatio-temporal simulation model can be applied to energy policy, if we regard the policy change group as "our side" and climate change as "the enemy" then what is critical is the speed of policy action compared with the speed of climate change combined with changes in variables that affect it such as population growth and economic development.

\section{Discussion and conclusions}

By DMSP/OLS global night-time light image data and sub-provincial energy consumption, population size, land area and other statistical data processed by inter-calibration, intra-annual integration and inter-annual correction, spatio-temporal geographically weighted regression models are constructed among stable night lightness DN value with per capita energy consumption and energy consumption per unit area. The provincial energy consumption in China is estimated with population size and land area based on the simulation. According to the estimation results, the simulated value of the model is close to the actual energy consumption with the goodness of fit over 99\%. In terms of the average annual energy consumption in 2000-2013, the relative errors of the annual average energy consumption in two models are both within 5.5\%, and the relative errors of all provinces are within $1 \%$. From both time and space perspectives, the relative errors in most provinces are within 5\%. 
The model estimation results indicate that the spatio-temporal simulations of China's energy consumption based on stable night-time light image data perform well, and can be used to estimate and forecast energy consumption in China's provinces as we;; as providing a complementary reference for monitoring and evaluating the energy consumption by satellite remote sensing data. With the improvement of global and Chinese satellite remote sensing technologies and applications, it becomes a future application direction to develop satellite image data services through "global-country-region" energy consumption monitoring. The key is to exploit the natural advantages of all days, wide coverage and objective accuracy of remote sensing satellite imagery data. By making full use of China's autonomous remote sensing satellite imagery data and foreign remote sensing satellite resources, satellite imagery data with other statistical databases involving population size and land area could be correlated, in order to format algorithms coupling the satellite image data and statistical data. Hence, energy consumption in China's provinces can be effectively and efficiently simulated, which provides data support for regional energy-consumption monitoring and assessment.

In the future, the key direction of China's provincial energy consumption forecast based on satellite night-time lighting data is to use machine learning techniques to extract effective information from satellite remote sensing data sources. Using daily, ten-day, monthly, quarterly, and annual satellite remote sensing data and energy consumption models to monitor energy consumption in national, provincial, municipal, district, and key areas dynamically and provide scientific decision support for macroeconomic trends.

The spatio-temporal simulation model of China's provincial energy consumption based on satellite night-time light data proposed in this paper can be widely applied to "three-dimensional" energy consumption spatio-temporal simulation at the global, national and regional levels. It provides a quick and accurate supplementary to the monitoring on the world's energy consumption pattern, changes in energy consumption of major economies, changes in energy consumption of country's internal regions. Research ideas also provide references for other similar studies involving carbon emissions, electricity consumption, economic growth, and urbanization monitoring and evaluation. 


\section{Acknowledgements}

This study was supported by the National Key R\&D Program of China (2016YFA0602601), Special Items Fund of Beijing Municipal Commission of Education, Beijing Social Science Fund, Program of Beijing Energy Development Research Center (NYJD20170101) and National Social Science Fund of China (15ZDA011).

\section{References}

[1] Mi Z, Meng J, Guan D, Shan Y, Song M, Wei Y-M, et al. Chinese CO2 emission flows have reversed since the global financial crisis. Nature communications. 2017;8:1712.

[2] Cong R G, Wei Y M. Potential impact of (CET) carbon emissions trading on China's power sector: A perspective from different allowance allocation options. Energy, 2010;35:3921-3931.

[3] Cong R G, Wei Y M. Experimental comparison of impact of auction format on carbon allowance market. Renewable \& Sustainable Energy Reviews, 2012;16:4148-4156.

[4] Mi Z, Zhang Y, Guan D, Shan Y, Liu Z, Cong R, et al. Consumption-based emission accounting for Chinese cities. Applied energy. 2016;184:1073-81.

[5] Liu Z, Guan D, Crawford-Brown D, Zhang Q, He K, Liu J. Energy policy: A low-carbon road map for China. Nature. 2013;500:143.

[6] Liu Z, Davis SJ, Feng K, Hubacek K, Liang S, Anadon LD, et al. Targeted opportunities to address the climate"Ctrade dilemma in China. Nature Climate Change. 2016;6:201.

[7] Mi Z, Meng J, Green F, Coffman DM, Guan D. China's "exported carbon" peak: patterns, drivers and implications. Geophysical Research Letters. 2018.

[8] Liu Z, Guan D, Wei W, Davis SJ, Ciais P, Bai J, et al. Reduced carbon emission estimates from fossil fuel combustion and cement production in China. Nature. 2015;524:335.

[9] Liu Z. National carbon emissions from the industry process: Production of glass, soda ash, ammonia, calcium carbide and alumina. Applied energy. 2016;166:239-44.

[10] Wei Y, Liu H, Song W, Yu B, Xiu C. Normalization of time series DMSP-OLS nighttime light images for urban growth analysis with pseudo invariant features. Landscape and Urban Planning. 2014;128:1-13.

[11] Liu Z, He C, Zhang Q, Huang Q, Yang Y. Extracting the dynamics of urban expansion in 
China using DMSP-OLS nighttime light data from 1992 to 2008. Landscape and Urban Planning. 2012;106:62-72.

[12] Lo C. Urban indicators of china from radiance-calibrated digital dmsp-ols nighttime images. Annals of the Association of American Geographers. 2002;92:225-40.

[13] Tan M. Urban growth and rural transition in China based on DMSP/OLS nighttime light data. Sustainability. 2015;7:8768-81.

[14] Zhang Q, Seto KC. Mapping urbanization dynamics at regional and global scales using multi-temporal DMSP/OLS nighttime light data. Remote Sensing of Environment. 2011;115:2320-9.

[15] Pandey B, Joshi PK, Seto KC. Monitoring urbanization dynamics in India using DMSP/OLS night time lights and SPOT-VGT data. International Journal of Applied Earth Observation \& Geoinformation. 2013;23:49-61.

[16] Wu J, Wang Z, Li W, Peng J. Exploring factors affecting the relationship between light consumption and GDP based on DMSP/OLS nighttime satellite imagery. Remote Sensing of Environment. 2013;134:111-9.

[17] Tang L, Cheng H, Qu G. Estimating Provincial Economic Development Level of China Using DMSP/OLS Nighttime Light Satellite Imagery. Advanced Materials Research. 2013;807-809:1903-8.

[18] Dai Z, Hu Y, Zhao G. The Suitability of Different Nighttime Light Data for GDP Estimation at Different Spatial Scales and Regional Levels. Sustainability. 2017;9:305.

[19] Qi K, Hu YN, Cheng C, Chen B. Transferability of Economy Estimation Based on DMSP/OLS Night-Time Light. Remote Sensing. 2017;9(8):786.

[20] Shi K, Chen Y, Yu B, Xu T, Chen Z, Liu R, et al. Modeling spatiotemporal $\mathrm{CO}_{2}$ (carbon dioxide) emission dynamics in China from DMSP-OLS nighttime stable light data using panel data analysis. Applied Energy. 2016;168:523-33.

[21] Zhang X, Wu J, Peng J, Cao Q. The Uncertainty of Nighttime Light Data in Estimating Carbon Dioxide Emissions in China: A Comparison between DMSP-OLS and NPP-VIIRS. Remote Sensing. 2017;9:797.

[22] Su Y, Chen X, Li Y, Liao J, Ye Y, Zhang H, et al. China's 19-year city-level carbon emissions of energy consumptions, driving forces and regionalized mitigation guidelines. Renewable \& 
Sustainable Energy Reviews. 2014;35:231-43.

[23] Meng L, Graus W, Worrell E, Huang B. Estimating CO2 (carbon dioxide) emissions at urban scales by DMSP/OLS (Defense Meteorological Satellite Program's Operational Linescan System) nighttime light imagery: Methodological challenges and a case study for China. Energy. 2014;71:468-78.

[24] Hara M, Okada S, Yagi H. Presumption of Carbon-Dioxide Emissions by Nighttime Lights Observed by DMSP/OLS -Presumption of Carbon-Dioxide Emissions Change in East Asian Region-(Understanding for each and integrated ecosystem using remote sensing, 6th International Symposium on Integrated Field Science). Journal of Integrated Field Science. 2009;6:162-.

[25] Oda T, Maksyutov S. A very high-resolution ( $1 \mathrm{~km} \mathrm{X} 1 \mathrm{~km}$ ) global fossil fuel $\mathrm{CO}_{2}$ emission inventory derived using a point source database and satellite observations of nighttime lights. Atmospheric Chemistry and Physics. 2011;11:543-56.

[26] Raupach M, Rayner P, Paget M. Regional variations in spatial structure of nightlights, population density and fossil-fuel $\mathrm{CO}_{2}$ emissions. Energy Policy. 2010;38:4756-64.

[27] Doll $\mathrm{CH}$, Muller J-P, Elvidge CD. Night-time imagery as a tool for global mapping of socioeconomic parameters and greenhouse gas emissions. AMBIO: a Journal of the Human Environment. 2000;29:157-62.

[28] Ghosh T, Elvidge CD, Sutton PC, Baugh KE, Ziskin D, Tuttle BT. Creating a Global Grid of Distributed Fossil Fuel $\mathrm{CO}_{2}$ Emissions from Nighttime Satellite Imagery. Energies. 2010;3:1895-913.

[29] Shi K, Chen Y, Yu B, Xu T, Yang C, Li L, et al. Detecting spatiotemporal dynamics of global electric power consumption using DMSP-OLS nighttime stable light data. Applied Energy. 2016;184:450-63.

[30] Welch R. Monitoring urban population and energy utilization patterns from satellite data. Remote Sensing of Environment. Remote Sensing of Environment. 1980;9:1-9.

[31] Elvidge CD, Baugh KE, Kihn EA, Kroehl HW, Davis ER, Davis CW. Relation between satellite observed visible-near infrared emissions, population, economic activity and electric power consumption. International Journal of Remote Sensing. 1997;18:1373-9.

[32] Chand TK, Badarinath K, Elvidge C, Tuttle B. Spatial characterization of electrical power consumption patterns over India using temporal DMSPCIOLS night@ltime satellite data. 
International Journal of Remote Sensing. 2009;30:647-61.

[33] Amaral S, Camara G, Monteiro AnMV, Quintanilha JA, Elvidge CD. Estimating population and energy consumption in Brazilian Amazonia using DMSP night-time satellite data. Computers Environment \& Urban Systems. 2005;29:179-95.

[34] Letu H, Hara M, Yagi H, Tana G. Estimating the energy consumption with nighttime city light from the DMSP/OLS imagery. Urban Remote Sensing Event2009. p. 1-7.

[35] Townsend AC, Bruce DA. The use of night-time lights satellite imagery as a measure of Australia's regional electricity consumption and population distribution. International Journal of Remote Sensing. 2010;31:4459-80.

[36] He C, Ma Q, Liu Z, Zhang Q. Modeling the spatiotemporal dynamics of electric power consumption in Mainland China using saturation-corrected DMSP/OLS nighttime stable light data. International Journal of Digital Earth. 2014;7:993-1014. 